thinnest ones fall very slowly. The thin pieces of glass are caught on the surface of a vessel filled with water. Afterwards they are taken up from this water surface on the silver support for the electron microscope. The water is sufficient for the adhesion of the glass layer to the support. It is better, however, to fix the glass layer at one point by means of 'Formvar' used as adhesive material. In Figs. 1 and 2 are reproduced pictures, taken with a Philips electron microscope (E.M. 100), of the same silver layer obtained by sputtering on glass and the other on 'Formvar'. The magnification is about 20,000 for each.

It can be seen that the pictures obtained are similar in first approximation and are composed of small grains on a continuous underlayer. However, the grains on glass seem to be somewhat larger, as is also the separation between them. The macroscopic thickness, evaluated by weighing a test-plate, was found to be $950 \mathrm{~A}$.

We are now trying to use much greater magnifications by making still thinner glass layers. At present the glass layers are made from Jena Geräte Glass 20.

We wish to thank the Centre Belge des Brasseries et des Malteries for financial help for this research.

Institute for Low Temperatures
A. VAN ITTERBEeK
L. De Greve
G. F. VAN VEELEN
C. A. F. TUYNMAN

and Technical Physics,

Louvain.

May 5.

\section{Dielectric Constant of Water Films}

IN order to explain some recent high-frequency (430 Mc./s.) measurements by Cownie and Palmer ${ }^{1}$ on the variation in the dielectric constant of moist clay with water content, it was assumed ${ }^{2}$ that the dielectric constant of the water increased exponentially from 3 (the high-frequency value for ice) at the water - clay boundary to 80 (the normal value for water) at an infinite distance from the boundary. It was supposed that the maximum change occurred in the thin layers of water close to the solid, and that the effects extended into the bulk of the liquid for appreciable distances.

That thin layers of water do exhibit abnormal mechanical and electrical properties is well known, and the terms 'bound' water ${ }^{3}$ or 'l'eau "solide" 'have been used to indicate the ice-like properties of thin water films. The restricted movement of such 'bound' water molecules would presumably result in a value of the dielectric constant less than the normal value of 80 .

In addition to the assumption that the dielectric constant of a water film varies with its thickness, it was found desirable to make a further assumption that the clay particles were both uniform in size and spherical in shape, although no steps had been taken to ensure this when the original experiments were undertaken. Consequently it has been decided to continue the investigation into the dielectric constant of thin water films by using certain composite or 'synthetic' dielectrics which are more amenable to theoretical treatment than is a clay - water mixture.

Preliminary experiments were made at frequencies of 2 and $3 \mathrm{Mc} / \mathrm{s}$. with thin mica plates separated by layers of water. It was found that the dielectric constant of the water decreased with the thinness of the film from more than 20 for films about $5 \mu$ in thickness to less than 10 for films about $2 \mu$ in thickness (thickness calculated assuming no absorption). Even with very wet composite dielectrics, the dielectric constant of the water did not exceed 60 .

It is $\mathrm{known}^{5}$ that the dielectric constant of ice at a temperature not very much below $0^{\circ} \mathrm{C}$. changes rapidly from about 90 to 3 when the frequency increases from just below to just above $10 \mathrm{kc} . / \mathrm{s}$. It was therefore thought that 'solid' water might exhibit a comparable variation about this frequency. To test this possibility, composite mica-water dielectrics were measured at $2 \mathrm{Mc} . / \mathrm{s}$. and.at $2 \cdot 5 \mathrm{kc} . / \mathrm{s}$. The value of the dielectric constant of the block of dielectric at the lower frequency was approximately double the value obtained at the higher frequency, indicating that the value of the dielectric constant of the inter-layer water was probably trebled or even quadrupled. In order to prevent evaporation between successive measurements, it was not possible (in these preliminary experiments) to make the necessary weighings which would enable the dielectric constant of the water alone to be calculated. A check experiment showed that the dielectric constant of the mica remained constant for this range of frequencies. Thus, since the dielectric constants of both water and mica are not frequency-sensitive over the range of frequencies used, whereas ice is frequency-sensitive at about $10 \mathrm{kc} . / \mathrm{s}$. , it would appear that the water film is tending to act not as 'solid' water but rather as 'liquid' ice.

This is perhaps a subtle distinction; but it is an important one when problems relating to the dielectric properties of thin water films are under consideration. It is also conceivable that this point of view may contribute to a better understanding of the abnormal mechanical properties of thin liquid films.

Further experiments are in progress to confirm these conclusions, and it is proposed to extend the investigation to various forms of 'synthetic' dielectrics with liquids other than water as the matrix. We are indebted to our colleague Mr. J. Muir for assistance with the lower-frequency measurements.

L. S. PALMER

A. Cunliffee

J. M. HougH

Physics Department,

University College of Hull. May 17.

${ }^{1}$ Cownie, A., and Palmer, L. S., Proc. Phys. Soc., B, 65, 295 (1952).

${ }^{2}$ Palmer, L. S., Proc. Phys. Soc., B, 65, 674 (1952).

${ }^{3}$ Blanchard, K. C., Cold Spring Harbor Symposium, No. 8, 1 (1940). Freymann, Marie, and Freymann, René, C.R. Acad. Sci., Paris, 232, 401 (1951).

'Dorsay, N. E., "The Properties of Common Water Substance", 105 (1940).

\section{Relations between Small-Angle Inter- ferences of Silk and the Wax Content}

IN previous papers ${ }^{1}$ it has been established that alkali treatment of fresh preparations of silk fibroin gives rise to the appearance of new equatorial smallangle reflexions which were believed to be higher orders of a ground-period of $45 \mathrm{~A}$. At first this effect was ascribed to a structural change of the silk lattice. Continued investigations, however, made it very likely that the new reflexions are due to some waxy substances. This assumption has now been confirmed 